\title{
Brain embolic infarction associated with cardiac amyloidosis in a patient with multiple myeloma: a bone marrow-heart-brain crosstalk
}

\author{
Jaeho Kim ${ }^{1}$, Jaehong Park ${ }^{1}$, Sung-Ji Park' ${ }^{2}$, Sung Mok Kim³ ${ }^{3}$ Kihyun Kim", \\ Jong-Won Chung ${ }^{1}$, Oh Young Bang
}

\begin{abstract}
${ }^{1}$ Department of Neurology, Samsung Medical Center, Sungkyunkwan University School of Medicine, Seoul, Korea
${ }^{2}$ Division of Cardiology, Department of Medicine, Samsung Medical Center, Sungkyunkwan University School of Medicine, Seoul, Korea

${ }^{3}$ Department of Radiology, Samsung Medical Center, Sungkyunkwan University School of Medicine, Seoul, Korea

${ }^{4}$ Division of Hematology-Oncology, Department of Medicine, Samsung Medical Center, Sungkyunkwan University School of Medicine, Seoul, Korea
\end{abstract}

Received: February 9, 2018

Revised: February 21, 2018

Accepted: February 22, 2018

Corresponding author:

Jaeho Kim

Department of Neurology,

Samsung Medical Center,

Sungkyunkwan University

School of Medicine, 81 Irwon-

ro, Gangnam-gu, Seoul 06351,

Korea

Tel: +82-2-3410-3599

E-mail: jaeho14.kim@samsung. com

This is an Open Access article distributed under the terms of the Creative Commons Attribution Non-Commercial License (http:// creativecommons.org/licenses/ by-nc/4.0/).

\section{ABSTRACT}

Cardiac amyloidosis is characterized by the extracellular fibril deposition of amyloid protein in the myocardium. Cerebral embolism caused by cardiac amyloidosis is rare. We report a case of 59-year-old woman with multiple myeloma who developed cerebral infarction probably related to cardiac involvement of amyloidosis, and discuss the pathophysiological mechanisms based on kidney biopsy and characteristic echocardiographic and cardiac magnetic resonance imaging features.

Keywords: Amyloidosis; Cardiomyopathies; Cerebral infarction; Magnetic resonance imaging; Multiple myeloma

\section{INTRODUCTION}

Immunoglobulin amyloid light chain amyloidosis may occur as a primary disease or in association with multiple myeloma or other plasma cell dyscrasia [1]. The heart is affected in over $50 \%$ of cases [2] but cerebral embolism in relation to cardiac amyloidosis has rarely been reported $[1,3]$. Herein, we report a patient with multiple myeloma who suffered from embolic infarction associated with cardiac amyloidosis diagnosed based on the kidney biopsy, echocardiographic and cardiac magnetic resonance imaging (MRI) results.

\section{CASE REPORT}

A 59-year-old woman suddenly developed vertigo type dizziness. Six years ago, she visited our hospital because of back pain. Spine MRI showed multiple spine mass and computed tomog- 



Fig. 1. (A) Fluid attenuated inversion recovery brain magnetic resonance imaging shows cerebral infarcts in the right posterior inferior cerebellar artery territory. (B) Magnetic resonance angiography reveals no vessel abnormality.
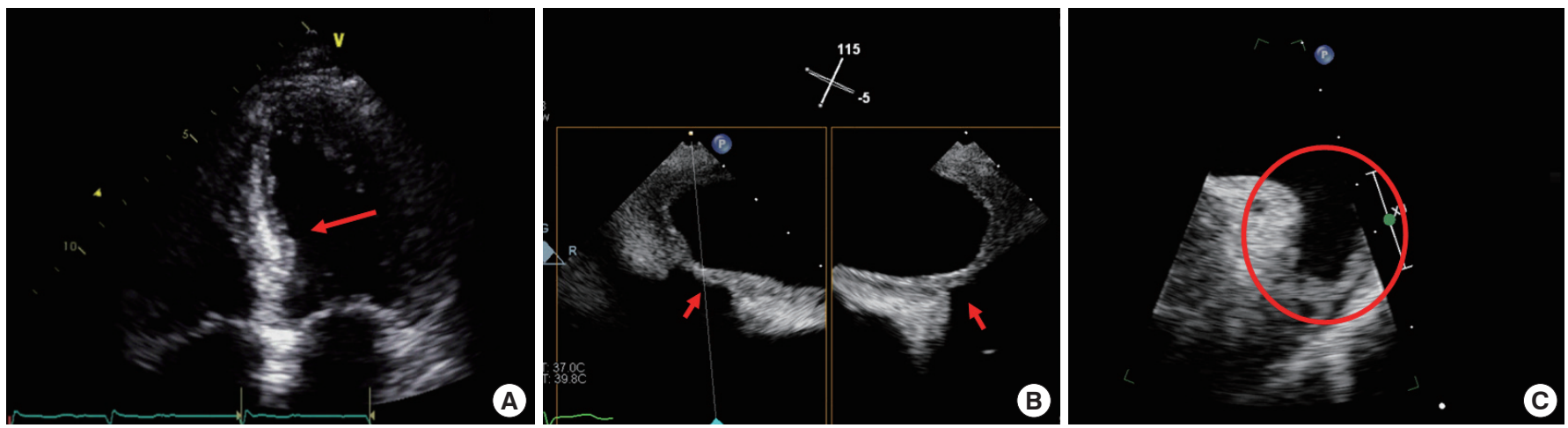

Fig. 2. Echocardiographic findings. (A) Increased left ventricle basal septal wall thickness (arrow), (B) left atrium (LA) wall thickness (arrows), (C) spontaneous echo contrast without thrombi in LA appendage (circle).

raphy (CT) guided bone biopsy revealed clusters of squeezed atypical cells. Laboratory test results showed hypercalcemia, elevated serum lambda $(\lambda)$ concentration $(4,980.00 \mathrm{mg} / \mathrm{L})$ and urine protein electrophoresis revealed suggestive of monoclonal proteinuria (M-protein 2,884.2 mg/day). Kidney biopsy, stained with Congo red, demonstrated amyloid deposition. Based on these findings, she was diagnosed as having multiple myeloma with amyloidosis. Thalidomide, cyclophosphamide, and dexamethasone were prescribed.

The patient was non-smoker and non-alcoholics. On neurologic examination, she showed right-sided ataxia in cerebellar function test and other neurologic examination was normal. Routine electrocardiography (ECG) and 24-hour Holter ECG monitoring revealed episodes of 2 nd degree atrioven- tricular block (Mobitz type I) and no atrial fibrillation or sustained atrial flutter. Laboratory test results showed no significant abnormality of thrombotic profile. Brain CT 12 hours after the onset showed no evidence of intracranial bleeding and brain MRI revealed acute infarcts in the right posterior inferior cerebellar artery (PICA) territories (Fig. 1A). Perfusion-weighted MRI showed perfusion delay related to the lesion and magnetic resonance (MR) angiography showed no evidence of vessel abnormality (Fig. 1B).

Transesophageal echocardiography showed symmetrical thickenings of the left ventricular (LV) and left atrial (LA) wall. LA was enlarged and transmitral Doppler showed diastolic dysfunction, and the cardiac involvement of amyloidosis was suspected (Fig. 2). There was spontaneous echo contrast 

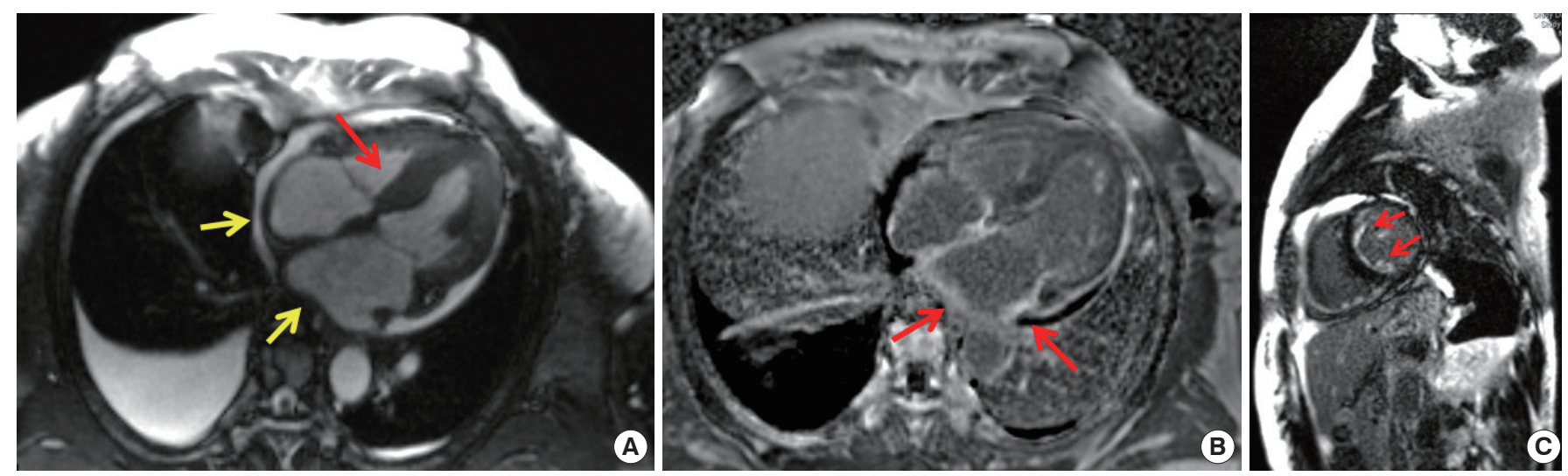

Fig. 3. (A) Cardiac magnetic resonance imaging showing increased left ventricle (LV) basal septal wall (red arrow) and atrial wall (yellow arrows) thickness, (B) left atrium wall enhancement, and (C) LV subendocardium enhancement.

(SEC) without thrombi in LA appendage (Fig. 2C).

In suspicion of cardiac amyloidosis, she underwent cardiac MRI which showed increased LV basal septal wall thickness and increased both atrial wall thickness. In late gadolinium enhancement imaging, myocardium showed suboptimal nulling in generally and LV subendocardial enhancement of LV myocardium and LA wall enhancement was prominent (Fig. 3). These findings were consistent with cardiac involvement of amyloidosis. The patient was treated with warfarin. She recovered completely 2 days after the onset.

\section{DISCUSSION}

This case illustrates an example of the bone marrow-heartbrain crosstalk. The patient had a cerebral infarct in the right PICA territory but no vascular risk factors or atherosclerotic changes in MR angiography suggesting that embolization was the etiology of her stroke. Cardiac amyloidosis was diagnosed by characteristic of echocardiographic and cardiac MRI findings and result of the kidney biopsy.

To the best of our knowledge, this is the first case which showed the cardioembolic stroke caused by cardiomyopathy related to amyloid deposition in patients with multiple myeloma with specific findings of cardiac MRI. Typical features of echocardiography of cardiomyopathy related to cardiac amyloidosis include thickened LV walls with normal to small cavity size, biatrial enlargement, thickened valves, and diastolic dysfunction [4,5]. In addition, typical cardiac MRI features of this condition are tissue characterization of the myocardium: transmural late gadolinium enhancement, LV wall thickness and biatrial enlargement, and pericardial effusion [5].

The mechanisms of embolization in amyloid cardiomyopa- thy are multifactorial $[1,3],(1)$ mural thrombosis due to blood stasis and intracavitary turbulence [6-8], (2) myocardial ischemic, endocardial damage [7], (3) arrhythmia (e.g., atrial fibrillation) may due to LA dilatation and failure with increasing wall stress $[9,10]$, and (4) plasma hypercoagulability state resulted from nephrotic syndrome, thrombocytosis related to hyposplenism, thrombin-antithrombin pathway impairment [11]. In this case, mural thrombosis due to cardiomyopathy was considered as the mechanisms of stroke, and prophylactic anticoagulation was indicated because SEC was observed in LA appendage.

Etiological workups are complex in patients without demonstrable arterial stenosis or potential sources of cardioembolism [12]. The case presented here demonstrated that multidisciplinary approach involving oncologist, cardiologist, and neurologist are important to evaluation and treatment of patients with ischemic stroke.

\section{CONFLICTS OF INTEREST}

No potential conflict of interest relevant to this article was reported.

\section{REFERENCES}

1. Zhang XD, Liu YX, Yan XW, Fang LG, Fang Q, Zhao DC, et al. Cerebral embolism secondary to cardiac amyloidosis: a case report and literature review. Exp Ther Med 2017;14: 6077-83.

2. Merlini G. CyBorD: stellar response rates in AL amyloidosis. Blood 2012;119:4343-5.

3. Cho KH, Cho YM, Kim JS. Embolic infarction associated with cardiac amyloidosis. J Clin Neurol 2005;1:92-6. 
4. Falk RH, Quarta CC. Echocardiography in cardiac amyloidosis. Heart Fail Rev 2015;20:125-31.

5. Mohty D, Damy T, Cosnay P, Echahidi N, Casset-Senon D, Virot $P$, et al. Cardiac amyloidosis: updates in diagnosis and management. Arch Cardiovasc Dis 2013;106:528-40.

6. Owa M, Takei Y, Hashikura Y, Kawasaki S, Koyama M, Ikeda S. Recurrent cerebral embolism in a familial amyloid polyneuropathy patient who received partial liver transplantation from a living donor. Intern Med 2001;40:25964.

7. Rice GP, Ebers GC, Newland F, Wysocki GP. Recurrent cerebral embolism in cardiac amyloidosis. Neurology 1981; 31:904-7.

8. Rahman JE, Helou EF, Gelzer-Bell R, Thompson RE, Kuo C,
Rodriguez ER, et al. Noninvasive diagnosis of biopsy-proven cardiac amyloidosis. J Am Coll Cardiol 2004;43:410-5.

9. Buja LM, Khoi NB, Roberts WC. Clinically significant cardiac amyloidosis. Clinicopathologic findings in 15 patients. Am J Cardiol 1970;26:394-405.

10. Dardas PS, Tsikaderis DD, Mezilis N, Geleris P, Boudoulas H. Echocardiographic evidence of atrial myopathy in amyloidosis: a case report. Eur J Echocardiogr 2002;3:303-5.

11. Sucker C, Hetzel GR, Grabensee B, Stockschlaeder M, Scharf RE. Amyloidosis and bleeding: pathophysiology, diagnosis, and therapy. Am J Kidney Dis 2006;47:947-55.

12. Bang OY, Ovbiagele B, Kim JS. Evaluation of cryptogenic stroke with advanced diagnostic techniques. Stroke 2014;45:1186-94. 